\title{
AVALIAÇÃO DO IMPACTO DA FRAGMENTAÇÃO DE FLORESTAS NATIVAS SOBRE A MESOFAUNA EDÁFICA NA REGIÃO DE DOURADOS-MS
}

\author{
Evaluation of impact of fragmentation of native forests about \\ the soil mesofauna Dourados-MS
}

\author{
Ana Amélia Gomes ${ }^{1}$,Rosilda Mara Mussury ${ }^{2}$, Silvana de Paula Quintão Scalon², Fabiana Watthier \\ Keilla Aparecida Almeida Cunha ${ }^{3}$, Homero Scalon Filho ${ }^{4}$
}

\begin{abstract}
RESUMO
Este trabalho, objetivou-se avaliar o impacto da fragmentação sobre a dinâmica populacional de indivíduos da mesofauna edáfica em três fragmentos de florestas nativas na região de Dourados, MS, sendo dois deles localizados em área indígena (Jaguapiru e Bororó) e o terceiro em outra área não indígena (fazenda Azulão). Para avaliar o impacto da fragmentação, foi avaliado o número de Acari Oribatida e Gamasida e insetos Collembola nas três áreas de estudo, utilizando o funil de Berlesse modificado. Observou-se que o fragmento Bororó apresentou um número médio total de 8,42 organismos seguido da Jaguapirú com 11,25 e Azulão com 16,74. Os Acari Oribatida e Gamasida foram os mais numerosos nas três áreas estudadas.
\end{abstract}

Termos para indexação: Acari, Collembola, mesofauna edáfica.

\begin{abstract}
This work aimed to evaluate the impact of fragmentation on populational dynamic of individual of soil mesofauna in three fragments of native forests in Dourados-MS, from which two of them are located inside indigenous area (Jaguapiru and Bororó) and the third in a non-indigenous area (Azulão farm). For evaluating the impact of fragmentation, number of Oribatid and Gamasida Acari and Collembola insects in these three areas of study was evaluated using a modified Berlesse funnel. It was observed that Bororó fragment presented a total average number of 8.42 organisms, Jaguapiru with 11.25 and Azulão with 16.74. Oribatida and Gamasida Acari were the most numerous organisms in both three studied areas.
\end{abstract}

Index terms: Acari, Collembola, mesofauna of soil.

(Recebido em 22 de junho de 2005 e aprovado em 11 de maio de 2006)

\section{INTRODUÇÃO}

Um dos grandes problemas ambientais do mundo moderno é a crescente fragmentação dos ecossistemas florestais que freqüentemente relaciona-se a efeitos deletérios sobre as comunidades bióticas (LAURANCE et al., 2002), como a redução de espécies vegetais e animais que, associada à pressão humana sobre os ecossistemas em paisagens fragmentadas, também contribui negativamente (TURNER, 1996). De acordo com Viana (1995) e Turner (1996), em regiões nas quais o processo da fragmentação se iniciou há muitas décadas, perturbações antrópicas constantes representam uma importante ameaça à biodiversidade.

As florestas proporcionam grandes benefícios ao solo, uma vez que reduzem a compactação e a erosão, mediante à atenuação progressiva do impacto da chuva, em virtude da existência de vários extratos na vegetação e da manta orgânica formada sobre o solo (SILVA et al., 1997).

Muitas florestas no Mato Grosso do Sul têm sido destruídas para implantação de campos agrícolas, sendo que, o uso do solo na agricultura após a retirada da vegetação natural, tem freqüentemente mostrado alterações em suas propriedades químicas e biológicas, as quais são dependentes das condições do solo, do clima, do tipo de cultura e das práticas culturais adotadas (MARCHIORI JR. \& MELO, 2000). De acordo com Gassen (2000), outro fator que é letal à população de organismos da mesofauna edáfica é a exposição do solo à radiação solar resultando na elevação da temperatura.

Constata-se na região de Dourados, MS que as terras demarcadas para os índios são insuficientes e mal distribuídas e que o arrendamento, tanto para produtores

\footnotetext{
'Especialista - Centro Universitário da Grande Dourados/UNIGRAN - Rua Balbina de Mattos, 2121, Jardim Universitário - $79800-000$ - Dourados, MS anaameliagomes@yahoo.com.br

2Doutora Faculdade de Ciências Biológicas e Ambientais - Faculdade de Ciências Agrárias - Universidade Federal da Grande Dourados/UFGD Rodovia Dourados-Itahum, Km 12 -79800-000 - Dourados, MS - mara.mussury@ufgd.edu.br; silvana.scalon@ufgd.edu.br

${ }^{3}$ Graduanda - Centro Universitário da Grande Dourados/UNIGRAN - Rua Balbina de Mattos, 2121, Jardim Universitário - $79800-000$ - Dourados, MS fabianawathier@bol.com.br; keillaacc@yahoo.com.br

${ }^{4}$ Mestre - Universidade Estadual de Mato Grosso do Sul/UEMS - Rodovia Aquidauana/CERA, Km 12, Cidade Universitária - $79800-000$ - Dourados, MS homero@uems.br
} 
como para outros indígenas, o desmatamento desordenado e o manejo inadequado do solo, têm provocado alterações ambientais e com isso extinguido importantes espécies vegetais para a reserva.

Os Acari Oribatida e Collembola por serem, os artrópodes de solo mais numerosos e melhor distribuídos influenciam diretamente na fertilidade do solo, por meio da estimulação da atividade microbiana, inibição de fungos e bactérias causadora de doenças, que contribuem para a formação do solo (BUTCHER \& SNIDER, 1971; THOMPSON \& EDWARDS, 1974; PRIMAVESI, 1990), fazendo o transporte de matéria orgânica, em avançado estado de decomposição, para níveis mais profundos do perfil do solo e vice-versa (HOLE, 1981; SAUTTER \& SANTOS, 1994). Contribuem ainda para a estrutura do solo criando um ambiente fértil e aumentando consideravelmente a porosidade do solo (BERG \& PAWLUK, 1984), aceleram a mineralização dos nutrientes (SEASTED \& CROSSLEY, 1980; SEASTED, 1984), bem como aumentam até seis vezes a velocidade de decomposição dos resíduos vegetais (BEHAN et al., 1978).

Vallejo et al. (1987) observaram nas matas secundárias a riqueza de matéria orgânica e que a proteção direta dos raios solares no solo favorece a abundância de organismos da mesofauna. Da mesma forma, Mussury et al. (2002) verificaram que as populações de Collembola aumentam com o aumento no teor de matéria orgânica, principalmente os organismos Entomobryidae, que de acordo com Sautter et al. (1996), são formas tipicamente epiedáficas, que encontrando grande disponibilidade de matéria orgânica na superfície do solo ocorrem em abundância.

Segundo Primavesi (1990), a lavração, a queimada, a exposição do solo ao sol e o uso de adubos amoniacais fazem com que a maioria da mesofauna desapareça.

O presente trabalho teve o objetivo de avaliar o impacto da fragmentação sobre a dinâmica populacional de indivíduos da mesofauna edáfica em três fragmentos de florestas nativas na região de Dourados, MS durante o período de março a dezembro de 2004.

\section{MATERIAL E MÉTODOS}

Este trabalho conduzido no Município de Dourados, localizado a $22^{\circ} 13^{\prime} 16^{\prime \prime}$ de latitude Sul e $54^{\circ} 48^{\prime} 2^{\prime \prime}$ de longitude Oeste e tem altitude de $452 \mathrm{~m}$. O clima é classificado como Cwa e a precipitação média anual é de $1500 \mathrm{~mm}$, com temperatura média anual de $22^{\circ} \mathrm{C}$. O solo da região é do tipo Latossolo Vermelho distroférrico (EMBRAPA, 1999), de textura argilosa e de topografia plana.
As coletas foram realizadas em três fragmentos de floresta. O fragmento Azulão (22 ${ }^{\circ} 11^{\prime} 57^{\prime}$ 'S e $54^{\circ} 54^{\prime} 29^{\prime \prime} \mathrm{W}$ ) é uma reserva de mata nativa de 54 ha na bacia do córrego Curral de Arame. Os outros dois fragmentos localizam-se respectivamente na reserva indígena Jaguapirú e Bororó (22¹0'22'’S e 5449'05'W). $\mathrm{O}$ fragmento Jaguapirú (aproximadamente 2 ha) localizase na bacia do córrego Sardinha e o fragmento Bororó (menor que 1 ha) localiza-se na bacia do córrego Laranja Doce. A vegetação dos três fragmentos é classificada como mata mesófila semi-decídua. Caracteriza-se pela mistura de espécies caducifólias e perenifólias. Os fragmentos aparentemente apresentam-se em vários estádios de degradação e regeneração. Especialmente os fragmentos localizados na Reserva Indígena apresentam-se mais degradados pela ação antrópica, como extração de madeira, principalmente de espécies vegetais do subdossel para obtenção de lenha e pelo impacto do cultivo de monoculturas, além do desmatamento que cada vez mais fragmenta os habitats remanescentes. Mesmo sendo uma área que também sofre com o impacto das lavouras de monocultura que a cerca, o fragmento Azulão parece ser a área melhor preservada devido sua maior extensão contínua de área de mata.

No período de março a dezembro de 2004, as coletas dos organismos da mesofauna edáfica foi realizada mensalmente em cada fragmento, sendo que, durante os meses de setembro e outubro não houve amostragens devido à proibição da FUNAI-Dourados, MS que impediu a circulação dos pesquisadores em áreas indígenas.

Os organismos da mesofauna edáfica foram coletados utilizando o funil de Berlesse modificado numa faixa de $0-5 \mathrm{~cm}$ de profundidade e com um volume de solo de $250 \mathrm{~cm}^{3}$ (BZUNECK \& SANTOS, 1991) em 10 pontos na área central dos fragmentos e em zigue-zague. As amostras foram acondicionadas em sacos plásticos para minimizar as perdas de umidade, e em seguida instaladas em mesas expositoras, cuja fonte de luz e calor foram lâmpadas de $25 \mathrm{w}$, permanecendo por sete dias no laboratório do Centro Universitário da Grande Dourados -UNIGRAN.

Os organismos foram recolhidos em potes plásticos, contendo solução de álcool 75\%,23\% de água destilada e $2 \%$ de glicerina. Após o período de exposição os organismos foram separados, identificados e contados com auxílio de um microscópio estereoscópio Lambda.

Os dados climáticos foram coletados na Estação meteorológica da Embrapa Agropecuária Oeste (Figura 1). 


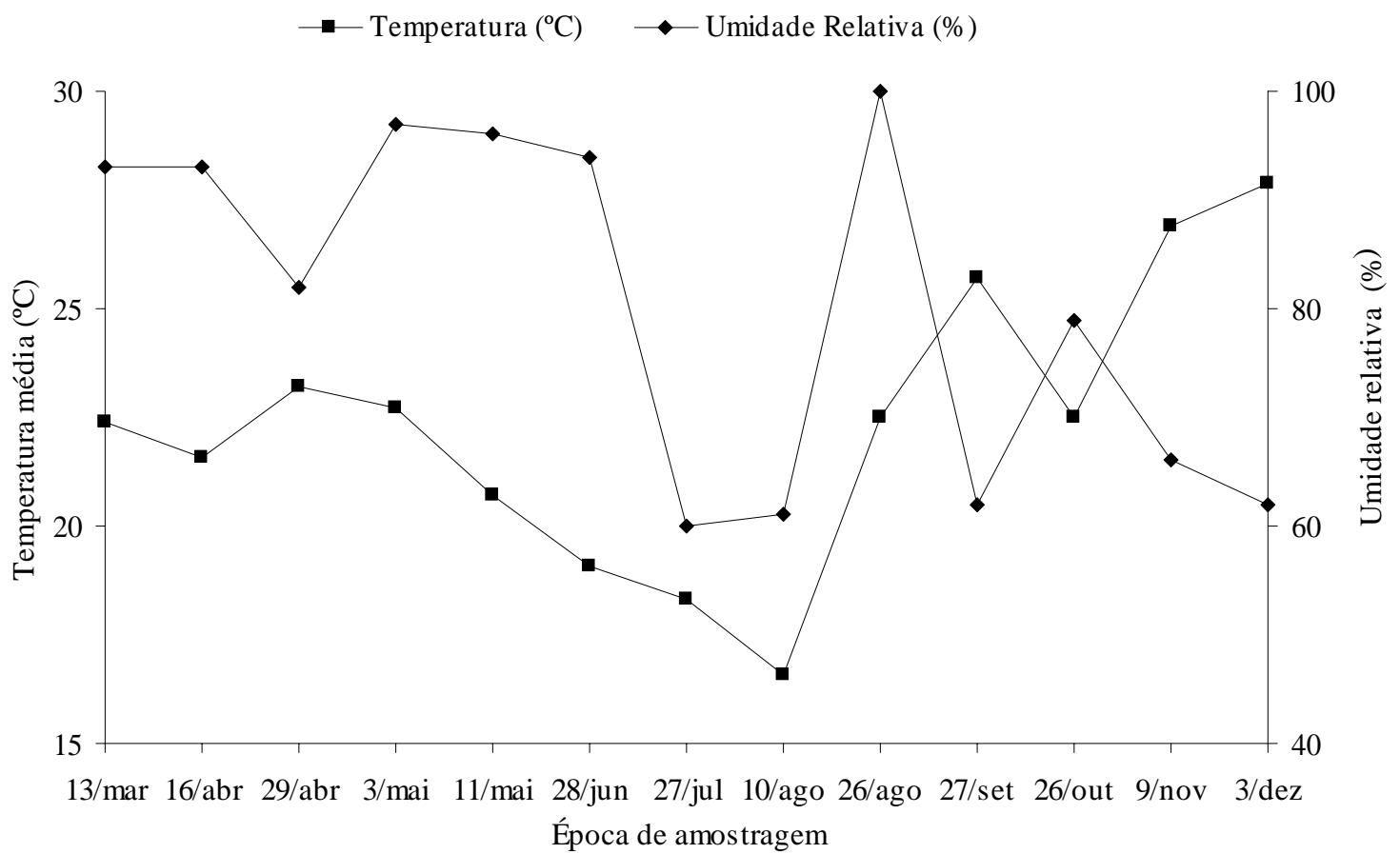

FIGURA 1 - Dados de temperatura e umidade relativa durante o período de estudo. (Fonte: Embrapa Agropecuária Oeste).

$\mathrm{O}$ experimento foi conduzido em delineamento inteiramente casualizado em esquema fatorial $3 \times 8 \times 5$, com 10 repetições. Os dados originais foram transformados para $\sqrt{\mathrm{x}+0,5}$ e as médias comparadas pelo teste Duncan a $1 \%$ de significância (SNEDECOR \& COCHRAN, 1989).

\section{RESULTADOS E DISCUSSÃO}

A análise de variância do número médio de organismos da mesofauna edáfica nas três áreas estudadas encontra-se na Tabela 1.

O número médio de organismos ocorrentes nos três fragmentos durante as oito épocas de amostragem está representado na Figura 2. Foram identificados Acari Oribatida e Gamasida e Collembola Entomobryomorpha. Devido ao inexpressivo número de Collembola Poduromorpha e Metaxypleona encontrados os dados não foram computados. Observou-se que o fragmento Bororó apresentou um número médio total de 8,42 organismos seguido da Jaguapirú com 11,25 e Azulão com 16,74.

Verifica-se que no fragmento Azulão e Jaguapiru que o número médio total de Acari e Collembola foram maiores do que no fragmento Bororó (Figuras 3 e 4 a, b, c).
Os fragmentos florestais estudados durante o período considerado, apresentam particularidades que nos permite relacionar a menor população de organismos da mesofauna edáfica encontrados. Provavelmente, o intenso pisoteio de animais e indígenas e a retirada de árvores no interior do fragmento, na reserva Bororó, observado in loco, decorre no afastamento dos troncos das árvores existentes, facilitando assim a movimentação de fatores bióticos e abióticos no interior do fragmento, associado ao deslocamento da massa de material orgânico (folha), expondo o solo. Conforme Richards (1974) e Primavesi (1990), a maioria dos animais edáficos são antifototrópicos, isto é não se adaptam adequadamente na presença de luz. Se o solo for desnudo, não há onde se refugiar e, portanto, morrem porque em seu estado despigmentado não suportam a insolação direta.

Conforme Bierregaard et al. (1992), as alterações nos ecossistemas levam a formação de fragmentos florestais de diferentes tamanhos e formas e podem resultar no isolamento de populações e até na extinção de espécies, reduzindo a biodiversidade local, em função, principalmente, de perda de habitats e de uma maior incidência de raios solares entre os fragmentos (WILCOX \& MURPHY, 1985). 
TABELA 1 - Dados da análise de variância da população de organismos da mesofauna edáfica considerando a época e o fragmento de coleta. Dourados - MS, 2004.

\begin{tabular}{cll}
\hline & \multicolumn{1}{c}{ Fontes de variação } & Valor de F \\
\hline \multirow{2}{*}{ Teste F } & Organismo & $8.8447 * *$ \\
& Época & $167.8592^{* *}$ \\
& Fragmento & $17.1718^{* *}$ \\
& Organismo X Época & $3.6864^{* *}$ \\
& Organismo X Fragmento & $5.3575^{* *}$ \\
& Época X Fragmento & $4.2148^{* *}$ \\
\hline $\mathrm{Cv}$ & Organismo X Época X Fragmento & $2.2562^{* *}$ \\
\hline
\end{tabular}

** Significativo a nível de $1 \%$ de probabilidade.

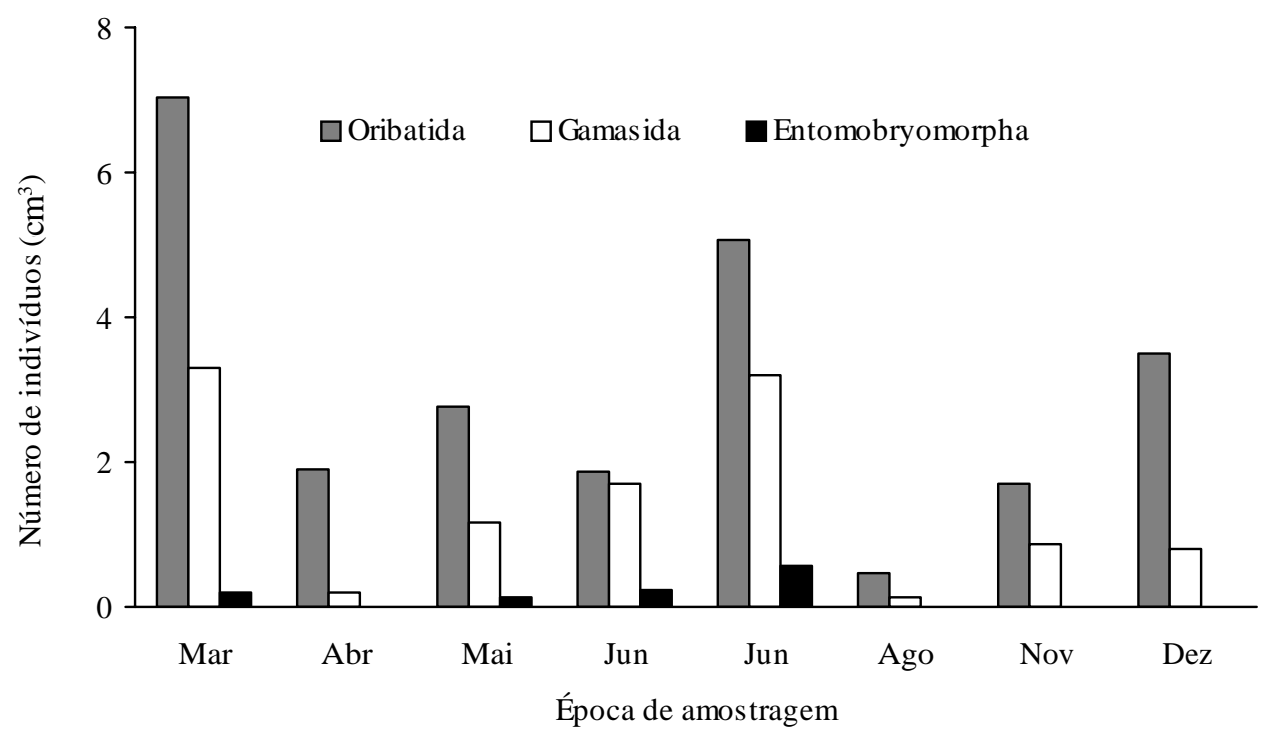

FIGURA 2 - Número médio de organismos ocorrentes nos três fragmentos, durante as oito épocas de amostragem. Dourados, MS. 2004.

Como os fragmentos Azulão e Jaguapiru são menos invadidos por animais, a vegetação no interior da mata ainda está mais adensada, e a superfície do solo está recoberta por folhas, provavelmente esses tenham sido os fatores que contribuíram para o maior número de organismos nessa área, quando comparado ao fragmento Bororó, pois conforme Sautter et al. (1996) e Vallejo et al. (1987), a abundância de matéria orgânica na superfície do solo favorece a diversidade biológica.
De acordo com Thomazini \& Thomazini (2000), na maioria dos casos relatados de fragmentação de florestas tropicais houve perda de espécies, entre elas os artrópodos, por meio, principalmente, da destruição do seu habitat; redução do tamanho da população; inibição ou redução da migração; eliminação de espécies dependentes de outras já extintas, entre outros fatores.

Os Acari Oribatida foram os organismos que ocorreram em maior número populacional, nas três áreas 
estudadas (Figura 3a e 4a, b, c). O número de Oribatida foi maior na fazenda Azulão, do que no fragmento Jaguapiru e Bororó principalmente nos meses de março e julho (Figura 4a, b, c). Observa-se que nas três áreas estudadas o número de Oribatida diferiu significativamente do número de Collembola quando essas estiveram presentes. Nos meses de maio, junho e julho na fazenda Azulão, não foram observadas diferenças significativas em relação ao número de Acari Gamasida, diferindo um pouco em relação aos outros dois fragmentos, isto é, no fragmento Jaguapiru nos meses de abril, maio e julho e no fragmento Bororó março, junho, julho e dezembro.

a)

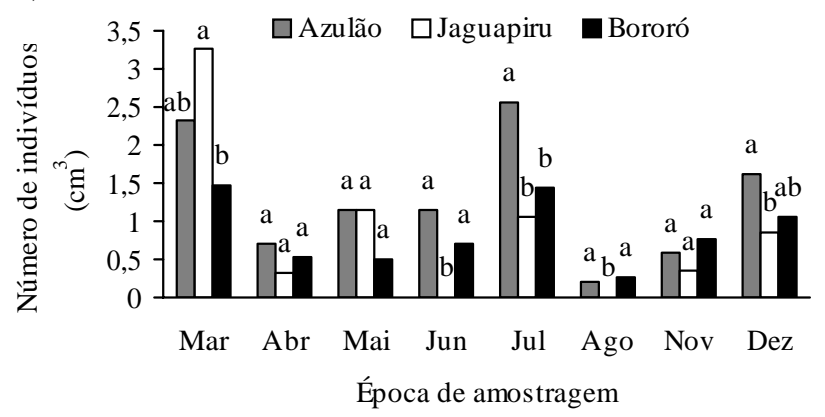

b)

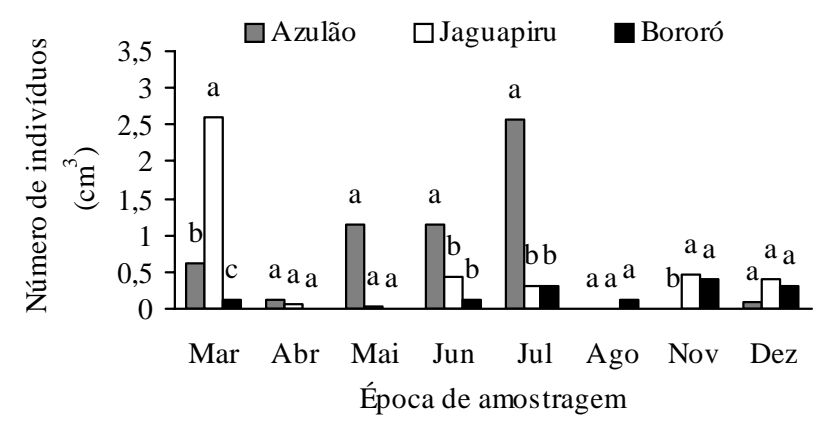

c)

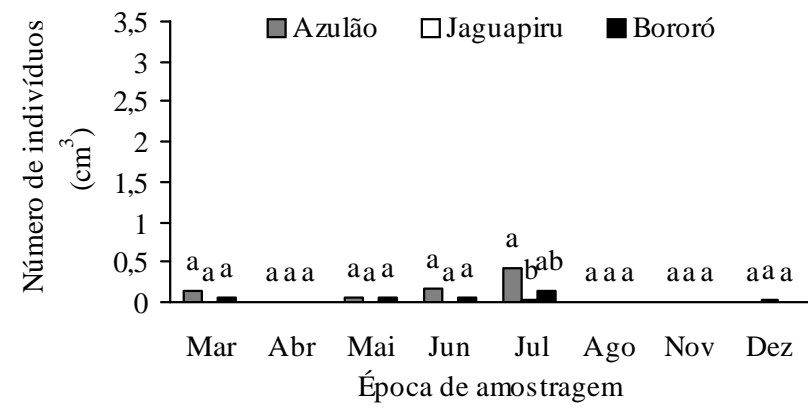

FIGURA 3 - Número médio de organismos Oribatida (a), Gamasida (b) e Entomobryomorpha (c), coletados nos três fragmentos de mata. Dourados-MS, 2004.
Mussury et al. (2002) observaram que em sistemas de cultivos com menor impacto sobre a fauna de solo as populações de Acari e Collembola desenvolvem-se, enquanto que no sistema de cultivo, onde havia muito revolvimento do solo, a densidade populacional de Collembola era menor. Segundo Tousignant \& Coderre (1992), Sauter \& Santos (1994), os Acari Oribatida em geral, demonstram uma maior preferência por habitats ricos em matéria orgânica, sendo os primeiros colonizadores de áreas perturbadas.

Acari Oribatida têm geralmente pouca capacidade para responder em curto prazo as alterações ambientais, suas populações declinam rapidamente quando os habitats são alterados, uma característica que pode permitir sua utilização para detectar a degradação ambiental (BEHANPELLETIER, 1999; SIEPEL, 1996).

Em todas as três áreas experimentais os Acari Gamasida ocorreram em menor quantidade do que o Oribatida, mesmo assim, pôde-se perceber que no fragmento Azulão e Jaguapiru a quantidade desse organismo foi maior que na Bororó. Nos meses de março e julho a população desses organismos ocorreram em maior densidade populacional (Figura $4 \mathrm{a}, \mathrm{b}, \mathrm{c}$ ).

De maneira geral, os Collembola ocorreram em menores índices populacionais quando comparados aos Acari. O fragmento Azulão foi o que apresentou maior número de organismos. Os Collembola Enthomobryomorpha ocorreram preferencialmente nos meses de março e julho, não ocorrendo nos meses de abril, agosto e novembro. Foram observados poucos organismos Poduromorpha e Metaxypleona.

Os organismos amostrados apresentaram reduções populacionais nos meses de abril e agosto, provavelmente devido às pequenas quedas de temperaturas apresentadas no período (Figura 1).

Os Collembola freqüentemente encontrados em solos de florestas nativas apresentaram uma redução expressiva, detectada no presente trabalho. Collembola encontradas nos solos em outras áreas da região, conforme relatado no trabalho de Mussury et al. (2002), não foram observadas no presente estudo, sendo que organismos Metaxypleona apenas um único exemplar foi encontrado. A explicação provável para o fato decorre da alteração no ecossistema provocada pela fragmentação, evento esse, que se dá em uma cascata de efeitos que podem culminar na extinção de espécies como observado por Myers, (1987); Bierregaard et al. (1992), além da destruição de habitats (TURNER, 1996). 
a)

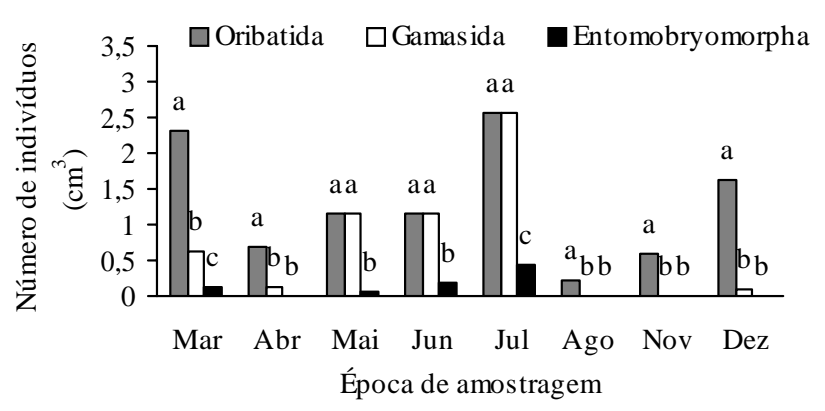

b)

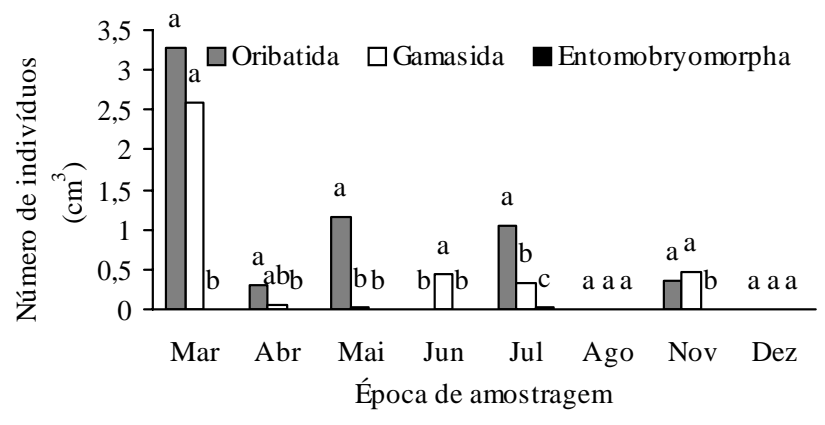

c)

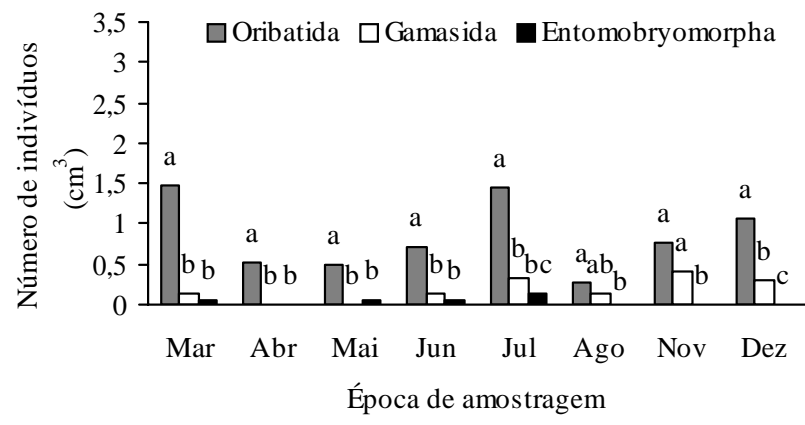

FIGURA 4-Número médio de organismos coletados nos fragmentos Azulão (a), Jaguapiru (b) e Bororó (c). Dourados-MS, 2004.

\section{CONCLUSÕES}

O fragmento da fazenda Azulão apresentou maior densidade populacional dos indivíduos da mesofauna edáfica por ser o fragmento menos "perturbado".

Os Acari Oribatida foram os mais numerosos nos três fragmentos avaliados
Nos meses de março e julho os Acari Oribatida e Gamasida e Collembola Entomobryomorpha ocorreram em maior abundância e no mês de agosto em menor abundância.

\section{AGRADECIMENTO}

À Fundação de Apoio ao Desenvolvimento do Ensino, Ciência e Tecnologia do Estado de Mato Grosso do Sul - FUNDECT, pelo apoio financeiro e ao Centro Universitário da Grande Dourados - UNIGRAN, pela oportunidade da realização do trabalho.

\section{REFERÊNCIAS BIBLIOGRÁFICAS}

BEHAN, V. A., STUART, B. B., McKEVAN, D. K. Effects of nitrogen fertilizes, as urea, on Acarina and other arthropods in Quebec black spruce humus. Pedobiologia, Jena, v.18, p. 249-263, 1978.

BEHAN-PELLETIER, V. M. Oribatid mite biodiversity in agroecoystems: role for bioindication. Agriculture, Ecosystem and environment, Amsterdam, v.74, p. 411-423, 1999.

BERG, N.W. ; PAWLUK, S. Soil mesofaunal studies under different vegetative regimes in north central Alberta. Canadian Journal Soil Science, Ottawa, v.64, p.209-223, 1984.

BIERREGAARD, R. O. LOVEJOY, T.E.; KAPOS, V.; SANTOS, A. A.; HUTCHINGS, W. The biological dynamics of tropical rainforest fragments. Bio Sciences, v.42, p. 859866, 1992.

BUTCHER, J.W. ; SNIDER, R.J.; Bioecology of edaphic Collêmbola and Acarina. Annual review of entomology. Palo Alto, v.16, p.249-288,1971.

BZUNECK, H.L. ; SANTOS, H.R. dos. Efeitos de dois Sistemas de Preparo do Solo e Sucessões de Cultura, na População de Ácaros Galumnidae (Cryptostigmata). Revista Ciência Agrária. v.11, n.1-2, 1991.

EMBRAPA, Centro Nacional de Pesquisas de solos. Sistema brasileiro de classificação de solos. Brasília:Embrapa Produção de Informação; Rio de Janeiro: Embrapa Solos. 412 p., 1999.

GASSEN, D.N. Os benefícios de corós em lavouras sob plantio-direto. Passo Fundo: EMBRAPA Trigo, 2000. 3p. (Embrapa Trigo. Comunicado Online, 47). 
HOLE, F.D. Effects of animals on soil. Geoderma, Amsterdam, v.25, p.75-112. 1981.

LAURANCE, W. F.; LOVEJOY, T. E.; VASCONCELOS, H. L.; BRUNA, E. M.; DIDHAM, R. K.; STOUFFER, P. C.; GASCON, C.; BIERREGAARD, R. O.; LAURANCE, S. G.; AND SAMPAIO, E. Ecosystem decay of Amazonian forest fragments: a 22-year investigation. Conservation Biology, Boston, v.13, n.3, p. 605-618, 2002.

MARCHIORI JUNIOR., M. ; MELO, W.J. Alterações na matéria orgânica e na biomassa microbiana em solo de mata natural submetido a diferentes manejos. Pesquisa Agropecuária Brasileira, Brasília, v.35, n.6, p.1177-1182, 2000.

MUSSURY, R. M.; SCALON, S. de P. Q.; SILVA, S. V., SOLIGO, R. V. Study of Acari and Collembolas population in four cultivation systems Dourados, MS. Brazilian Archives of Biology and Technology, Curitiba, v. 45, n. 3, p. 257-263, 2002.

MYERS, N. The extinction spasm impending: synergisms at work. Conservation Biology, v.1, n.1, p.14-21, 1987.

PRIMAVESI, A. Manejo ecológico do solo: Agricultura em regiões tropicais. $9^{a}$ ed. São Paulo: Nobel. 1990, p.142154

RICHARDS, B. N. Introduction to the soil ecosystem. Longmam Group Limited. New York, 1974. p.266.

SAUTER, K D.; KOBIYAMA, M.; USHIWATA. Influência do lodo de esgoto doméstico e lodo de água sobre a mesofauna edáfica. Arquivos de Biologia e Tecnologia, Curitiba, v.39, n.4, p. 745-750. 1996.

SAUTER, K. D. ; SANTOS, H. R. dos. Avaliação da estrutura da população da mesofauna edáfica, em diferentes regimes de reabilitação de um solo degradado pela mineração do xisto. Revista Ciência Agrárias, Curitiba, v.13, n.1-2, p. 31-34. 1994.

EASTED, T.R. The role of microarthropods in decomposition and mineralization processes. Annual review of entomology, Palo Alto, v.29, p.25-46, 1984.SEASTED, T.R. ; CROSSLEY, D. A. Effects of microarthropods on the seasonal dynamics of nutrients in forests littes. Soil Biology Biochemistry. Oxford, v.12, p.337-342, 1980.

SNEDECOR, G. W. ; COCHRAN, G. W. Statistical Methods. Ed. Lowa: State University Press ( $8^{\mathrm{a}}$ ed.), 1989, p.491. SILVA, M. L; VALVERDE, S. R; PASSOS, C. A. M. \& COUTO, L. Viabilidade do reflorestamento do eucalipto consorciado com a cultura do feijoeiro um estudo de caso. Revista Árvore, Viçosa, v.21, n.4, p.527-535, 1997.

SIEPEL, H. The importance of unpredictable and shortterm environmental extremes for biodivresity in oribatid mites. Biodiversity Letters, Oxford, v.3, p.26-34, 1996.

THOMAZINI, M. J. ; THOMAZINI, A.P.B.W. A fragmentação florestal e a diversidade de insetos nas floresta tropicais úmidas. Embrapa Acre, doc.57, 2000.

THOMPSON, A. R. ; EDWARDS, C. A. Effects of pesticides on nontarget invertebrates in freshwater and soil. In: GUENZI, W.D. (Ed.) Pesticides in soil and water. Madison: Soil Science Society of America. p. 341-375, 1974.

TOUSIGNANT, S.; CODERRE, D. Niche partitioning by soil mites in a recent hardwood plantation in Southern Quebec, Canada. Pedobiologia, Jena, v.36, p. 287-294, 1992.TURNER, I. M. Species loss in fragments of tropical rain forests: a review of the evidence. Journal Applied Ecology, v. 33, p. 200-209, 1996.

VALLEJO, L. R. ; FONSECA, C. L. da; GONÇALVES, D. R. P. Estudo comparativo da mesofauna do solo entre áreas de Eucaliptus citriodora e mata secundária heterogênea. Revista Brasileira de Biologia, Rio de Janeiro, v.47, n.3, p.363-70, 1987.

VIANA, V. M. Conservação da biodiversidade de fragmentos florestais em paisagens tropicais intensamente cultivadas. In: Abordagens interdisciplinares para a conservação da biodiversidade biológica e dinâmica do uso da terra, Belo Horizonte, p. 135-154, 1995.

WILCOX, B. A.; MURPHY D. D. Conservation estrategy: the effects of the fragmentation on extinction. The American Naturalist, v.125, p. 879-887, 1985. 